\title{
Application of active learning modalities to achieve medical genetics competencies and their learning outcome assessments
}

This article was published in the following Dove Press journal:

Advances in Medical Education and Practice

\author{
Nobuko Hagiwara \\ Division of Cardiovascular Medicine, \\ Department of Internal Medicine, \\ School of Medicine, University of \\ California, Davis, CA, USA
}

\begin{abstract}
The steadily falling costs of genome sequencing, coupled with the growing number of genetic tests with proven clinical validity, have made the use of genetic testing more common in clinical practice. This development has necessitated nongeneticist physicians, especially primary care physicians, to become more responsible for assessing genetic risks for their patients. Providing undergraduate medical students a solid foundation in genomic medicine, therefore, has become all the more important to ensure the readiness of future physicians in applying genomic medicine to their patient care. In order to further enhance the effectiveness of instructing practical skills in medical genetics, the emphasis of active learning modules in genetics curriculum at medical schools has increased in recent years. This is because of the general acceptance of a better efficacy of active learner-centered pedagogy over passive lecturer-centered pedagogy. However, an objective standard to evaluate students' skill levels in genomic medicine achieved by active learning is currently missing. Recently, entrustable professional activities (EPAs) in genomic medicine have been proposed as a framework for developing physician competencies in genomic medicine. EPAs in genomic medicine provide a convenient guideline for not only developing genomic medicine curriculum but also assessing students' competency levels in practicing genomic medicine. In this review, the efficacy of different types of active learning modules reported for medical genetics curricula is discussed using EPAs in genomic medicine as a common evaluation standard for modules' learning outcomes. The utility of the EPAs in genomic medicine for designing active learning modules in undergraduate medical genetics curricula is also discussed.
\end{abstract}

Keywords: undergraduate medical genetics education, genomic medicine competencies, active learning modules, entrustable professional activities, learning outcome assessment

\section{Introduction}

Recent advancements in medical genetics and genomic medicine have resulted in a significant expansion of their application in day-to-day clinical practice. Accordingly, genetic conditions recognized and treated in present-day clinics are no longer limited to rare monogenic diseases. The translational application of genomic medicine to a wide range of pathogenic conditions has also been significantly accelerated in part by the rapidly decreasing costs of sequencing technologies. Therefore, the need for health professionals to have competence in medical genetics will only intensify in the future. In addition, public interest toward genetic/genomic testing and personalized medicine is steadily rising owing to direct-to-consumer genetic testing, a situation that will require physicians to be prepared to adequately answer patients' inquiries regarding genomic medicine. ${ }^{1,2}$ Currently, the demand for genetic services is growing, and
Correspondence: Nobuko Hagiwara Division of Cardiovascular Medicine, Department of Internal Medicine, School of Medicine, University of California, One Shields Avenue, Davis, CA 95616, USA

Tel +I 5302179733

Fax +I 5307547167

Email nhagiwara@ucdavis.edu 
physicians without formal training in medical genetics are more likely to be placed in the position of evaluating patients with genetic conditions because of the shortage of medical geneticist physicians and genetic counselors. ${ }^{3-5}$

Among these nongeneticist physicians, especially primary care physicians hold an important position in managing patients with genetic conditions; therefore, providing educational opportunities in genetics to primary care physicians is crucial. ${ }^{6,7}$ Indeed, it is becoming more common for primary care physicians to order or refer patients for cancer susceptibility testing. ${ }^{8}$ In addition, as the number of genetic conditions covered by state-mandated newborn screening programs is increasing, more and more primary care physicians will face difficulties in managing lifelong genetic conditions such as cystic fibrosis detected by newborn screening. ${ }^{9}$ Specialist physicians are equally in need of possessing knowledge and skills for identifying and managing genetic conditions relevant to their fields; for example, oncology and cardiology are at the forefront of applying genomic medicine-based diagnosis and treatments to patients. ${ }^{10,11}$

Despite the need for nongeneticist physicians to have literacy and basic skills in genetic/genomic medicine, the physician's readiness reported in literature consistently indicates that the majority of practicing physicians do not feel well prepared for applying genomic information to their patient care. ${ }^{12,13}$ Reflecting this trend, there have been reports of negative impact on patient care due to the use of genetic testing by nongeneticist physicians. ${ }^{14-16}$ The reports of underutilization of genetic services by primary care physicians are also noted, suggesting that a lack of familiarity with and perceived barriers to genetic services are hindering primary care physicians from using genetic testing. ${ }^{17,18}$

In response to the rapidly growing demand for all physicians to have basic skills in genetic/genomic medicines, multiple medical science education organizations have developed competencies in genetic and genomic medicines that practicing physicians should possess. Among them are guidelines for undergraduate medical genetics education. Briefly, these include genetics educational objectives presented by the Association of American Medical Colleges' Medical School Objective Project (AAMC MSOP; Contemporary Issues in Medicine: Genetics Education) in 2004, ${ }^{19}$ genetics competencies for medical school education proposed as part of AAMC-Howard Hughes Medical Institute (HHMI) Scientific Foundations for Future Physicians in $2009,{ }^{20}$ and a detailed list of medical genetics competencies for Medical School Core Curriculum in Genetics developed by the Association of Professors of Human and Medical Genetics
(APHMG) in 2013. ${ }^{21}$ The main goal of these publications has been to promote the development of curricula in medical genetics that focus on competencies rather than mere acquisition of knowledge. ${ }^{22}$ For example, the APHMG classified the list of genetics competencies into six categories in medical practice: patient care, medical knowledge, practice-based learning and improvement, professionalism, interpersonal and communication skills, and system-based practice, which are based on the residency program competencies set forth by the Accreditation Council for Graduate Medical Education (ACGME).

Following these developments, a new framework for physician education in genomic medicine based on "entrustable professional activities" (EPAs) has been proposed by the Inter-Society Coordinating Committee for Physician Education in Genomics (ISCC) consisting of National Institutes of Health (NIH) agencies and professional medical societies. ${ }^{23,24}$ EPAs are "units of professional practice, defined as tasks or responsibilities to be entrusted to the unsupervised execution by a trainee once he or she has attained sufficient specific competence" and allow supervisors to assess their trainees in daily clinical practice. ${ }^{25}$ Using this developing concept, five EPAs in genomic medicine have been developed by the ISCC: 1) family history; 2) genomic testing; 3 ) treatment based on genomic results; 4) somatic genomics; and 5) microbial genomics. ${ }^{23,24}$ The genomic medicine skills enlisted in each of these five EPAs are further subcategorized into eight competencies (Table 1). ${ }^{23}$ These competencies are based on the six core competencies identified by ACGME and the two new competencies recently added by the AAMC. ${ }^{26}$ The EPAs in genomic medicine and their embedded competencies are designed to be used as a flexible template for developing genomic medicine training programs tailored to the needs of individual medical specialties ${ }^{23}$ and, therefore, provide an excellent guide for developing undergraduate medical genetics courses.

The eight competencies of EPAs in genomic medicine are composed of well-defined genomic medicine skills specific to each EPA. For example, the "patient care" competency of the "family history" EPA includes "assemble family history" and "recognize patterns of Mendelian inheritance and calculate simple Mendelian risks." In contrast, the "patient care" competency of the genomic testing EPA includes "discuss the indications for genomic testing" and "order, interpret, and communicate the results of appropriate genomic tests." ${ }^{23}$ Using the EPAs in genomic medicine as a guide, genetics course materials of active learning modalities can be designed to provide well-focused training to select genomic medicine 
Table I Categories of EPAs in genomic medicine ${ }^{23}$

\begin{tabular}{|c|c|}
\hline EPA categories & Descriptions \\
\hline Family history & Elicit, document, and act on relevant family history pertinent to the patient's clinical status \\
\hline Genomic testing & Use genomic testing to guide patient management \\
\hline $\begin{array}{l}\text { Patient treatment based } \\
\text { on genomic results }\end{array}$ & Use genomic information to make treatment decisions \\
\hline Somatic genomics & $\begin{array}{l}\text { Use genomic information to guide the diagnosis and management of cancer and other disorders involving somatic } \\
\text { genetic changes }\end{array}$ \\
\hline $\begin{array}{l}\text { Microbial genomic } \\
\text { information }\end{array}$ & $\begin{array}{l}\text { Use genomic tests that identify microbial contributors to human health and disease as well as genomic tests that guide } \\
\text { therapeutics in infectious diseases }\end{array}$ \\
\hline \multicolumn{2}{|c|}{$\begin{array}{l}\text { Note: Each EPA contain the following eight core competencies, each of which is tailored to the specific EPA: patient care, knowledge for practice, practice-based learning and } \\
\text { improvement, interpersonal and communication skills, professionalism, systems-based practice, interprofessional collaboration, and personal and professional development. } \\
\text { Adapted by permission from Macmillan Publishers Ltd: Genetics in Medicine, copyright 20I4. Korf BR, Berry AB, Limson M, et al. Framework for development of physician } \\
\text { competencies in genomic medicine: report of the Competencies Working Group of the Inter-Society Coordinating Committee for Physician Education in Genomics. Genet } \\
\text { Med. } 2014 ; 16(1 \mathrm{I}): 804-809 .{ }^{23}\end{array}$} \\
\hline
\end{tabular}

competencies suited for each developmental stage of medical students. In addition, these EPAs provide a dependable standard for evaluating the efficacy of genetics curriculum when used to assess learning outcomes of the students.

In recent years, medical genetics educators across the nation have been integrating more clinical materials into their medical genetics curriculum. ${ }^{27}$ In order to increase student's learning efficiency in the clinical application of knowledge, medical genetics curricula are following the trend of increasing the use of active learning modalities. ${ }^{28}$ Although there is a large body of literature reporting the use of active learning modalities in medical genetics curricula, an absence of common assessment tools makes it challenging to compare the learning outcomes of these active learning courses. As outlined earlier, the EPAs in genomic medicine would provide not only a useful guideline for designing learning objectives for active learning modules but also an objective standard for assessing learning outcomes of the participating students. An EPA-based assessment would provide well-defined measurement of student's skill level in genomic medicine and proficiency in caring for patients with genetic conditions.

In this review, as an attempt to use the EPAs in genomic medicine as a common tool for outcome assessment, recent illustrative literature on active learning courses in undergraduate medical genetics curricula is reviewed through the lens of EPAs. Of note, three EPAs in genomic medicine, family history, genomic testing, and patient treatment based on genomic results, are mainly discussed because of the nature of instructional materials in undergraduate medical genetics courses.

\section{Types of active learning modalities for genomic medicine: applications and outcome assessments}

Simulations are becoming one of the core instruction modalities in medical education since they provide training toward achieving clinical competencies. ${ }^{29-31}$ The means for simulation-based exercises are diverse, ranging from highly technical applications involving a programmable simulator mannequin or computer-based virtual reality to nontechnical applications using methods such as a situation-based roleplaying, standardized patients, or case-based, problem-based learning. ${ }^{30}$ Because simulation exercises are based on active learning involving student's participation, it has been reported that the retention of the acquired knowledge by simulation exercises is better than traditional didactic teaching, ${ }^{30,32}$ leading to their widespread adoption as mainstream pedagogical methods in medical school curricula. ${ }^{33}$ Because of the enhanced learning efficacy of active learning pedagogy, ${ }^{34,35}$ the emphasis of learner-centered instruction in medical genetics curricula is also increasing. ${ }^{27}$ The main challenge we are currently facing is how to measure the effectiveness of an active learning genetics curriculum for achieving practical genomic medicine skills. Below, the recently published illustrative examples of learner-centered/simulation-based medical genetics courses are discussed using the EPAs in genomic medicine described in the "Introduction" section. Summaries of the active learning modalities discussed in the text (their advantages, disadvantages, and efficacies) are provided in Table 2.

Table 2 Summary of the active learning modalities for genomic medicine discussed in the text

\section{Role-playing}

Advantages - Students can learn to apply their newly acquired knowledge in a safe environment.

- The scenario of a case for role-playing is easily adjustable.

- Students can experience different roles in the clinical scenario.

- It is cost effective. 
Table 2 (Continued)

\begin{tabular}{ll}
\hline Role-playing & \\
\hline Disadvantages & - The activity may lack the sense of a real clinical \\
& situation. \\
- It requires multiple well trained facilitators to make & the learning activity successful. \\
Efficacies & - Learning to document relevant family history and \\
& conduct genetic risk assessment. \\
& - Developing interpersonal and communication skills \\
& collaborations \\
- Understanding the ethical ramifications of genetic & test results. \\
- Learning the importance of counseling patients with \\
genetic conditions.
\end{tabular}

Standardized patients

\begin{tabular}{|c|c|}
\hline Advantages & $\begin{array}{l}\text { - Well-trained standardized patients can create an } \\
\text { authentic learning atmosphere. } \\
\text { - Students can learn to apply their newly acquired } \\
\text { knowledge in a safe environment. } \\
\text { - The clinical scenario is adjustable. }\end{array}$ \\
\hline Disadvantages & $\begin{array}{l}\text { - Hiring enough well-trained standardized patients } \\
\text { could be costly. } \\
\text { - Since the standardized patient session is generally } \\
\text { one-on-one, it is hard to accommodate a large class } \\
\text { size. }\end{array}$ \\
\hline Efficacies & $\begin{array}{l}\text { - Learning to document relevant family history and } \\
\text { conduct genetic risk assessment. } \\
\text { - Developing interpersonal and communication skills. } \\
\text { - Understanding the ethical ramifications of genetic } \\
\text { test results. } \\
\text { - Learning firsthand the importance of counseling } \\
\text { patients with genetic conditions. }\end{array}$ \\
\hline \multicolumn{2}{|c|}{ Computer-based modules } \\
\hline Advantages & $\begin{array}{l}\text { - There is no physical constraint in designing a virtual } \\
\text { scenario, therefore it is highly adjustable to the } \\
\text { rapidly advancing genomic medicine. } \\
\text { - The learning format affords flexible scheduling and } \\
\text { pacing for learners. } \\
\text { - It can target a large number of students with } \\
\text { different skill levels } \\
\text { - It is cost effective. }\end{array}$ \\
\hline Disadvantages & $\begin{array}{l}\text { - It lacks the human encounter } \\
\text { - It can lack the sense of a real clinical situation. }\end{array}$ \\
\hline Efficacies & $\begin{array}{l}\text { - Acquisition of technical knowledge in genetic testing. } \\
\text { - Recognizing the ethical ramifications of genetic test } \\
\text { results. } \\
\text { - Cultivating an attitude for life-long learning }\end{array}$ \\
\hline
\end{tabular}

Case-based/problem-based learning

Advantages - It can highlight the clinical relevance of basic science in disease diagnosis and patient care.

- Designing cases for problem-based learning is highly adjustable.

- It can highly motivate students since the learning process is led by students.

Disadvantages - Group size needs to be relatively small.

- It requires multiple well-trained facilitators to make the session effective.
Table 2 (Continued)

\begin{tabular}{ll}
\hline Case-based/problem-based learning \\
\hline Efficacies & Learning to apply basic genetics concepts to clinical \\
& cases. \\
- Acquisition of technical knowledge in genetic testing. & Learning to document relevant family history and \\
recognize patterns of Mendelian inheritance. \\
- Cultivating an attitude for life-long learning
\end{tabular}

Personal genomic testing and cadaver genomic testing

Personal genomic testing

Advantages - The use of personal genomic information motivates students to learn.

- Practical skills to use genomic testing in patient care are obtained through in depth hands-on learning.

- An extensive array of knowledge for practicing genomic medicine can be obtained.

Disadvantages - Positive test results and sequence variants of unknown significance can cause psychologically adverse effects on students.

- Positive test results can cause family issues.

- Hands-on instruction is labor intensive on instructors and not applicable for a large class size.

- Genomic testing is expensive.

Efficacies - Gaining an in-depth understanding of the risks, benefits, and limitations of genomic testing.

- Learning to recognize pathogenic sequence variants and their inheritance pattern.

- Increasing confidence in the ability to advise patients on genomic test results.

- Gaining an in-depth understanding of genomic medicine concepts.

- Cultivating empathy towards patients with genetic conditions.

- Understanding the ethical ramifications of genetic test results.

- Cultivating an attitude for life-long learning.

Cadaver Genomic Testing

Advantages - There is no concern for psychologically adverse effects on participating students.

- Students are motivated to learn because of their invested work in cadaver dissection.

- Students gain an experience in clinical reasoning of genomic testing results in relation to the pathological findings of the cadaver.

Disadvantages - There could be potential ethical issues such as postmortem disclosure of genetic information.

- DNA sample preparation from cadaver for sequencing can be technically challenging.

- Genomic testing is expensive.

Efficacies - Gaining an understanding of the risks, benefits, and limitations of genomic testing in relation to the known pathological conditions.

- Learning to recognize pathogenic sequence variants and their inheritance pattern.

- Learning to recognize that genomic tests require interpretation with respect to the pathogenic condition.

- Understanding the ethical ramifications of genetic test results

- Cultivating an attitude for life-long learning 


\section{Role-playing}

Role-playing has been used as an effective method for training personnel in many different disciplines ${ }^{36}$ including medical students..$^{37,38}$ Role-playing simulates a scenario-based environment allowing students to gain new perspectives from reflection on the scenario and also to learn to apply their newly acquired knowledge and skills to a simulated situation. This learning method is cost-effective and highly learner centered because, by design, students are placed in simulated environments and perform problem-solving activities. ${ }^{39}$ Role-playing is highly effective in training students to develop interpersonal and communication skills across different disciplines. ${ }^{40-42}$ Because of these attributes, role-playing has been applied to provide exercises to medical students to gain genetic counseling skills..$^{43,44}$

In a small-size pilot study $(\mathrm{N}=15-17)$ conducted at the University of Alabama at Birmingham, first-year medical student volunteers were asked to play the role of a counselee in Mendelian disease vignette-based mock sessions conducted by genetic counselors. The following three genetic conditions were used for the mock sessions: hereditary breast and ovarian cancer syndrome, fragile $\mathrm{X}$ syndrome, and autosomal recessive retinitis pigmentosa. This supplemental session was run in parallel with the mandatory medical genetics course. ${ }^{43}$ A comparable number of nonparticipating students were used as a control group to assess the outcome of the supplemental session. By administering a self-reported questionnaire before and after the role-playing sessions, the authors reported that the participating students gained an improved understanding of genetic testing, the importance of referrals for genetic counseling, the impact of genetic test results on patient's emotional and practical health care, and the role of genetic counselors in patient management. ${ }^{43}$ These subjects overlap with the important genomic medicine skills listed in the family history and genomic testing EPAs, including patient care, knowledge for practice, system-based practice, interpersonal and communication skills, and interprofessional collaborations competencies, ${ }^{23}$ therefore providing an effective learning experience for students to gain confidence in a wide range of skills required for the application of genetic information to practice. In the vignette-based counseling scenario, students learned to assemble family history, recognize patterns of Mendelian inheritance and estimate disease risks, choose appropriate genetic testing, interpret the test results, and discuss the test results with patients. In addition, the students in this course played the role of patients who are cared for by nonphysician health professionals (a genetic counselor), which offered an excellent opportunity to gain an appreciation for interprofessional patient care and develop professionalism in handling genetic cases through interprofessional collaboration. Although some participating students commented that the counseling session did not seem real, it appeared that by adjusting the scenarios and also the style of facilitators, the session could run more realistically. ${ }^{43}$ Owing to the small sample size, a quantitative analysis of the role-playing sessions was not performed in this study.

In a significantly large-scale study $(\mathrm{N}=2,326)$ conducted at the Third Military Medical University in Chongqin, China, the effectiveness of role-playing on gaining genetic counseling skills was assessed for third-year medical students. These students participated in a genetic counseling program offered as an integral part of the medical genetics course. ${ }^{44}$ The goal of this program was to help students learn to apply their knowledge in genetics to clinical problems, develop communication skills, and further promote their interest in genetics. The participating students were divided into small self-study groups and assigned a Mendelian disease clinical vignette; the roles of both counselor and counselee were played by students in the presence of a tutor. To assess the effectiveness of the role-playing session, students' attitudes toward the course and the mastery of 42 key genetics concepts in performing genetic counseling were evaluated using self-reported posttraining survey questionnaires (taken by $\sim 300$ randomly selected students) and pre- and postsession written examinations (taken by 200 randomly selected students), respectively. ${ }^{44}$ The survey results indicated that the program was well received, and $~ 80 \%$ of students agreed that role-playing helped them to 1) understand about genetic counseling; 2) master the knowledge of medical genetics by self-study; and 3) improve their communication skills. ${ }^{44}$ These results suggest that, as the University of Alabama study did, role-playing is an effective training method for students to obtain the core competencies of the family history and genomic testing EPAs, which are critical for genetic counseling. ${ }^{23}$

As the authors pointed out, the lack of students' evaluation based on their role-playing performance limits this study's outcome measurement to the acquisition of knowledge measured by test scores. Although performance-based assessment is technically challenging, doing so would significantly support the learning outcome and efficacy of role-playing as an active learning method in medical genetics. The authors of this study also noted the amount of effort required to train the teaching faculty; all 20 tutors recruited for the role-playing sessions underwent a significant amount of training and evaluation to become qualified, ${ }^{44}$ indicating the importance 
of a faculty development program in providing authentic and effective learning environments for role-playing sessions.

\section{Standardized patients}

The use of standardized patients (SPs) is a widely applied active learning method in health professionals' education. ${ }^{45-47}$ Although the basic premise of a hypothetical clinical scenario is shared with role-playing, well-trained standardized patients can make the encounter more realistic. ${ }^{41}$ Literature detailing the use of standardized patients for medical genetics curriculum is scarce; however, there is one report providing a detailed documentation of the use of SPs for undergraduate medical genetics education. ${ }^{48}$

This study was conducted at the Mount Sinai School of Medicine using cohorts of third-year medical students (a total of 136) who were partaking in the internal medicine clerkship. The goal of this study was to evaluate the effectiveness of an SP program in increasing medical students' competence in assessing genetic risks and communicating genetic test results and its ramification to patients. ${ }^{48}$ Students had two encounters with an SP portraying a woman in her 30s with a family history of breast cancer. In the first counseling session, students were expected to obtain the relevant family history, draw a three-generation pedigree, interpret the patient's risk, and counsel the patient for genetic testing. In the second session with the same SP several weeks later, students were instructed to communicate the positive genetic test result of a BRCA1 mutation and discuss its ramification for the patient and her family. ${ }^{48}$ To conduct successful counseling sessions in this scenario, students needed to collect relevant family history and evaluate cancer risks, communicate the ramifications of the family history, discuss the indication for genetic testing and make recommendations, and interpret and explain the test result to the patient. These tasks embody the genomic medicine competencies listed in the patient care, knowledge for practice, interpersonal and communication skills, and professionalism of the family history and genomic testing EPAs. ${ }^{23}$

In this study, students were evaluated in four ways: 1) Likert-scale self-assessments (1-5) measuring students' confidence levels in performing genetic counseling skills; 2) the SP's assessment of students' communication skills during the counseling session; 3) assessment of video-recorded students' counseling performance by a trained observer; and 4) grading of pedigrees drawn by students using an eight-question knowledge test. The self-assessment scores were used to measure students' improvements in the confidence toward genetic counseling by comparing between SP program-participating and nonparticipating (control) students and also by longitudinally comparing within the participating students before and after the sessions. ${ }^{48}$ Compared to the control students, students who participated in the SP program reported significantly higher confident scores in skills such as drawing a three-generation pedigree, assessing genetic risks for cancer based on family history, and communicating the genetic risk to a patient. In addition, longitudinally, students' confidence in assessing and communicating genetic risks increased significantly after the second counseling session. ${ }^{48}$ These observations indicate that the use of an SP is effective for students to obtain competence in genetic counseling. Similar to the reports for role-playing discussed earlier, an evaluation of students' improvement in their skill levels after the SP encounter was not conducted in this study. Activitybased assessment would be extremely valuable to tangibly measure the effect of an SP program on the student's mastery of genomic medicine skill sets.

Comparing role-playing and SP programs, the former is more flexible in format, allowing students to experience different roles (eg, physician, genetic counselor, and patient), and therefore, it could provide learning experiences in the interprofessional collaboration and development of empathy for patients. An often encountered challenge in role-playing is to generate an authentic atmosphere. ${ }^{43}$ An SP program, however, is more formulated to provide a close-to-real patient encounter for students, thus creating a more authentic learning environment. However, since it requires well-trained actors, it is rather costly to be used regularly. Therefore, combining these two programs, such as using role-playing for training students and SP for the assessment of students' competency levels, could create a robust genomic medicine active learning course. In a learner-centered medical genetics course designed as such, the EPAs in genomic medicine would provide an effective blueprint to design a program and an objective standard for measuring the mastery of clinical medical genetics skills acquired by students.

\section{Computer-based modules}

Computer-based instruction could be either software- or Internet-based modules and provides interactive and selfdirected learning environment for students. By combining clinical scenarios and digital simulation technologies, these modules can offer virtual clinical situations for learners. ${ }^{30}$ In addition, these modules can afford flexible scheduling and pacing for learners and also target a large number of students with different skill levels. Because of these merits, 
computer-based instruction can be broadly applied, including being used as a supplement for the core curriculum or as a segment of flipped classroom instruction. Currently, various online-based medical genetics courses, both free and proprietary, are available. For example, the G2C2 website (Genetics/Genomics Competency Center; http://genomicseducation.net/) lists educational resources categorized by topics (eg, clinical genetics and genetic counseling) and also indicates applicable genomic medicine competencies for each resource. Several of these online modules have been developed or maintained by publicly funded organizations such as the NIH.

In one report, interactive web-based learning modules funded by the National Human Genome Research Institute (NHGRI) were investigated for their effectiveness in supporting medical students to learn ethical, legal, and social implication (ELSI) issues in medical genetics. ${ }^{49}$ This webbased curriculum was developed following genetics core competency guidelines of several professional organizations (eg, American Society of Human Genetics and American Academy of Family Physicians) and aimed at helping learners develop knowledge and competence in genetic testing and counseling as well as encouraging changes in attitudes toward ELSI issues surrounding genetic testing and counseling.

Contents of these online modules were designed to be approachable by the first-year medical students and contained five topics such as pediatric genetics, newborn screening, prenatal genetic testing, breast and ovarian cancer risk assessment, and inheritance of complex disorders (some of these modules are available at www.larasig.com/geneticsactivity). Each learning module consisted of identical pre- and postmultiple-choice examinations, learning objectives, information presented in a text form, patient interview videos (some modules), and intermittent quizzes. A total of 897 medical students ranging from first year to fourth year participated in this study (the number of students who took each module varied from 163 to 596 ).

The knowledge gain supported by each module was quantified by comparing the pre- and postcompletion test scores. ${ }^{49}$ For all five modules, the average test scores showed statistically significant improvements (average scores for five modules combined were $54 \%$ correct for pretest and $70 \%$ correct for posttest). Changes in the attitude, selfefficacy, and intended behavior related to the ELSI were assessed by self-reported 5-point Likert-scale responses $(1=$ strongly disagree to $5=$ strongly agree $)$ to the statements relevant to each module. This self-reported assessment was administered after the completion of the module as a form of pre-/postretrospective test. In all five modules, each of these three ELSI categories showed a statistically significant increase.

Similar to the role-playing and SP programs discussed earlier, actions described in these self-reported statements encompass the patient care, knowledge for practice, interpersonal and communication skills, and professionalism competencies defined in the family history and genomic testing EPAs. ${ }^{23}$ This study shows the promises of interactive online modules in providing support for students to begin gaining practical skills in performing basic genetic counseling. Online modules, therefore, could be used as a cost-effective introductory training module preceding the performance-based training such as role-playing and SP program. In this manner, students' knowledge and skills acquired through online learning can be reinforced and evaluated by their physical performance. The outcome assessments obtained from students' physical performance can be then effectively applied to revise and improve the online modules.

A more recent report describes the creation of a virtual learning environment by linking a virtual cytogenetic laboratory and a virtual patient case for genetic counseling. ${ }^{50}$ Medical genetics education curriculum is constantly facing the challenge of keeping up with the rapidly advancing field of genomic medicine. The authors propose that the use of digital simulation technology in a classroom would be a cost-effective way to keep up with the rapid pace of developments in genetic testing technology and its application to clinical practice. The objectives of this study were to assess the effectiveness of the virtual reality learning module to improve 1) understanding of the translation of molecular genetics laboratory analysis to clinical practice; 2) knowledge of medical genetics; 3) intrinsic motivation for learning medical genetics; and 4) self-efficacy for performing medical genetics activities such as genetic counseling.

The learning module was developed by combining a virtual case of a pregnant couple whose fetus had a possible chromosomal abnormality, a virtual laboratory to conduct cytogenetic testing, and virtual genetic counseling of the couple based on the test results. ${ }^{50}$ A total of 300 first-year undergraduate students were enrolled, $84 \%$ of whom had major in medicine. The study did not contain control group students. Identical pre- and postintervention survey questionnaires (5-point Likert scale) were used to assess the students' motivation in learning medical genetics and self-efficacy in performing counseling. Multiple-choice examinations were administered to assess improvement in students' knowledge 
in cytogenetics. ${ }^{50}$ Since this study did not assess student learning outcomes based on competency, it is hard to evaluate the results in the framework of the EPAs in genomic medicine and competencies. However, the fact that $78 \%$ of the enrolled students reported that they felt more confident in counseling a patient after the session indicates that the virtual simulation exercise was effective to provide training in genetic counseling skills that are part of the competencies of the family history and genomic testing EPAs ${ }^{23}$ In addition, $\sim 90 \%$ of students found conducting cytogenetic examination in the virtual laboratory helped them to better understand medical genetics. In fact, the multiple-choice examination scores for the cytogenetics knowledge showed a significant improvement after the virtual simulation session, showing that the students' self-assessment accurately reflected a quantifiable gain in ability. ${ }^{50}$ The authors also noted that the self-motivation and self-efficacy were improved by the session, indicating that the virtual simulation experience would cultivate the attribute for lifelong learning of genomic medicine.

Virtual simulation allows the merging of physically separated environments into one scenario and shows robust promise to be a highly effective modality to train medical students in a wide range of medical genomics competencies. To evaluate outcomes of the online-learning modules in a real-life setting, role-playing or standardized patients could be used in conjunction with the EPAs in genomic medicine to provide a reliable standard for assessing students' competence levels in clinical genetics.

\section{Case-based/problem-based learning}

Problem-based or case-based learning is self-directed by students to solve the clinical mystery of a "real-world" patient case in an informative and peer-supported environment emphasizing teamwork. This learning modality is effective to highlight the relevance of basic science in diagnosis and patient management. ${ }^{51}$ At the Emory School of Medicine, this active learning modality was applied to an introductory module in medical genetics to create "virtual diagnostic laboratory" sessions. In these sessions, deidentified patients' genetic test results and laboratory reports from the clinical diagnostic laboratories were used as cases to discuss the application of genetic testing in common genetic disorders, which are most likely encountered by primary care physicians. ${ }^{52}$ The sessions were attended by the entire class of first-year medical students $(\sim 140)$, who were divided into groups of $15-16$ students. ${ }^{52}$ To bring clinical simulation into the classroom discussions, the authors developed four genetic disease scenarios depicting genetic disciplines including biochemical genetics, cytogenetics, molecular genetics, and cancer genetics. In these case discussion sessions, with the guidance of a facilitator, students were in charge of deciphering differential diagnosis of the disease, an appropriate genetic testing strategy, interpretation of the test results based on laboratory reports, risk assessment of the patient's family, and dissemination of the test result. To work on these problems, students were given web resources such as GeneTests and OMIM for self-directed learning.

The objectives of this course were that students would be able to 1) identify relevant and up-to-date information to support genetic testing decisions; 2) propose appropriate testing strategies for simple genetic cases; 3 ) interpret test results; and 4) communicate the test results to patients. The authors noted that this learning module addressed several of the APHMG medical genetics competencies (published before the genomic medicine EPAs).$^{52}$ From the standpoint of assessing the outcomes of active learning modules, however, the genomic medicine EPAs are more convenient to be used as a benchmark to evaluate students' mastery of genomic medicine skills because of their concise organization. In fact, the learning objectives of the virtual diagnostic laboratory sessions are fully concordant with the competencies listed in the patient care, knowledge for practice, interpersonal and communication skills, and personal and professional development competencies of the family history and genomic testing EPAs. ${ }^{23}$

The effects of the case-/problem-based sessions on students' performance improvement were not assessed in this report. Instead, student evaluations were collected from a randomly selected pool of $\sim 25$ students; $92 \%$ of them agreed or strongly agreed to the educational value of the virtual laboratory sessions, $88 \%$ agreed or strongly agreed to the suitability of the teaching format, and $94 \%$ agreed or strongly agreed that the teaching materials were useful and made the presentation more effective. Based on the student evaluations, the authors concluded that these sessions successfully underscored the utility of genetics to clinical medicine and were effective for students to learn to apply their knowledge in genetics to clinical cases. ${ }^{52}$ Since these sessions were developed as an anchor module of the vertically integrated genetics and genomics curriculum, ${ }^{52}$ it would be of great interest to assess the effectiveness of these sessions on students' performance in their clinical clerkship years.

\section{Personal genomic testing and cadaver genomic testing}

The steady increase in the application of whole exome sequencing (WES) and whole genome sequencing (WGS) to 
clinical diagnosis is intensifying the need of physicians to have literacies in genomic testing. In addition, public interests in personalized medicine and availability of direct-to-consumer genomic testing are increasing the chance for physicians to be asked by their patients about personalized preventative care. ${ }^{53}$ In order to provide the necessary knowledge and practical skills to use genomic testing, incorporation of personal genomic testing has been recently piloted in medical genetics courses. ${ }^{54,55}$ The benefits of using personal genomic testing for instruction include providing students an effective handson learning environment and motivating students to learn by offering an opportunity to work on their own genome. ${ }^{56}$

So far two institutions, namely, Stanford School of Medicine and Icahn School of Medicine at Mount Sinai (ISMMS), have reported on the application of personalized genomic testing (PGT) in the form of an elective medical genomics course. ${ }^{57,58}$ Genome-wide single nucleotide polymorphism (SNP) array was used by the Stanford course, and WGS was used by the ISMMS course. The course participants included medical students, graduate students, residents, and fellows.

The Stanford study reported the results of 46 participants. ${ }^{57}$ The students first took a 2-week in-depth instruction on PGT and then were offered to have their own genomic DNA genotyped by a direct-to-consumer genetic testing company or, instead, use publicly available data sets for a hands-on training in genomic data analysis. The study was designed to assess changes in the attitudes and knowledge about genomics and PGT as measured using surveys and the objective knowledge improvements in genomics and PGT as measured using written examinations. The surveys and written examinations were administered before and after the PGT session for comparison. The obtained data were compared longitudinally within the groups (students who chose personal genotyping "genotyped" - and students who chose public data base "nongenotyped") and also between the two groups to assess the effectiveness of the hands-on genomics session and the use of personal genomic testing. The survey results indicated that the genotyped students gained better understanding of the risks and benefits in using PGT and interpretation of PGT results compared to the nongenotyped students. ${ }^{57}$ Although the effect was less, the nongenotyped students still reported that the course increased their knowledge in genetics and their understanding of PGT results, suggesting that hands-on learning of genomics using public database is still effective for students to gain genomics skills. The genotyped students also developed higher levels of empathy toward patients with genetic conditions; $100 \%$ of the genotyped students reported that the course helped them to understand the experience of patients who undergo PGT, while only half of the nongenotyped students indicated as such. ${ }^{57}$ The objective knowledge assessments by written tests showed that although the presession test scores were comparable between the genotyped and nongenotyped groups, the postsession test scores of the genotyped group were significantly higher than those of the nongenotyped group. ${ }^{57}$

It is interesting to note that even though a majority of the students in this study commented that the given opportunity to ask health care professionals for help in interpreting the PGT test results was an important component of this course, few of them actually did (13\%) or were planning to ask $(13 \%) .{ }^{57}$ In the companion article,${ }^{59}$ it was described that the students felt that they were well equipped to interpret the PGT results; therefore, they rarely utilized genetic counseling service even though it was offered for free. This observation may suggest that to cultivate a receptive attitude toward interprofessional collaboration in genomic testing, further encouragement and structured interprofessional activity built into the course may be necessary.

The Mount Sinai study reported the results of 19 participants. ${ }^{58}$ Before the genomic testing session was offered, the participating students took the introductory genomics course, which provided thorough information on PGT. ${ }^{60}$ The genomic testing course offered hands-on bioinformatic analysis of raw genomic sequence data, which could be done using student's personal genome or one in public databases. In the present study, all participating students chose to sequence their own genome; therefore, only longitudinal analysis was reported. ${ }^{58}$ In order to assess the impact of using the personal WGS, written examinations and self-reporting questionnaires were administered at the selected time points, such as immediately before and after the course and 6 months after the completion of the course ( 6 months follow-up). The questions were designed to evaluate 1) technical knowledge and understanding of WGS; 2) attitudes toward personal WGS; 3 ) perceived utility of personal genome analysis; and 4) psychological impact of personal genomic information (eg, depression, anxiety, and regret). In addition, as part of a 6-month follow-up, in-depth phone interviews were conducted. ${ }^{58}$

The examination scores assessing the technical understanding of WGS significantly improved after the session compared to before the session. Self-reported understanding of WGS assessed by qualitative questionnaires (1-5-point scale) showed the highest average point at the 6-month follow-up, suggesting that students' self-learning continued after the course. Negative psychological effects were reported 
by two participants, which were caused by a sequence variant associated with schizophrenia and a sequence variant associated with Brugada syndrome. The authors noted that the positive educational outcome of PGT could be eroded if students are distressed by unanticipated test result. The analysis of in-depth 6-month follow-up interviews indicated that the use of personal genome sequence significantly increased motivation for learning genomic analysis and developed more empathy for the patient's experience. Interestingly, underutilization of genetic counseling service was also observed in the Mount Sinai study, despite the fact that the service was provided for free. At the 6-month follow-up time point, no students reported having discussed their results with a genetic counselor. ${ }^{58}$

Genomic medicine competencies that can be attained by the application of PGT are extensive. The practical skill training offered in a PGT course encompasses all eight competency categories represented in the EPAs in genomic medicine, namely, family history, genomic testing, and patient treatment based on genomic results. ${ }^{23}$ To name a few, these include 1) learning to recognize pathogenic sequence variants, their inheritance pattern (both Mendelian and multifactorial), ramification of the test result, ethical issues of genomic testing, self-directed learning, and genomic datadriven treatment options and 2) learning to communicate all the relevant information to the patients. In the two studies discussed earlier, there is no doubt that participants gained significant knowledge and confidence in understanding the benefits and risks of genomic testing and correctly interpreting the test results. However, no consistent assessment tool was used to evaluate the learning outcomes in the context of genomic medicine competency. Indeed, the authors of the Mount Sinai study noted "consensus on what domains of knowledge are necessary for future health care providers and other users of personal genomics, and how best to measure these domains, is lacking. ${ }^{58}$ Because of its flexible nature and easy to follow format, the EPAs in genomic medicine could provide the consensus necessary for designing the PGT courses and the standard for measuring the learning outcomes of participating students. Since genomic medicine is a rapidly moving field, computer-based interacting modules such as virtual simulation discussed earlier might be good evaluation tools for testing students' mastery of genomic medicine skills acquired by the use of PGT.

When PGT is used in the classroom, the possibility of incidental findings and detection of variants of unknown significance always exists, and the obtained genetic information could have a significant impact on the student and members of the student's family. To circumvent the anticipated negative effects associated with the use of personal genomic data and still maintain students' self-motivation to learn genomic medicine, the use of cadaver WES has been implemented at Temple University School of Medicine. ${ }^{61,62}$ In this course, the entire class of first-year medical students (MS1s) (total of 210) was organized into teams of six students and the cadavers to be sequenced were selected based on the cause of death and pathological findings. ${ }^{62}$ Since students invested significant hours in dissecting the cadavers, it was postulated that a strong incentive existed for students to uncover the genetic basis of the cadaver's pathogenic conditions.

Because of the large class size, hands-on bioinformatics analysis was not offered to the students. Instead, whole exome sequences of cadaver DNA were obtained using a commercial service. The students were provided with the preselected clinically relevant single-nucleotide variants (SNVs; eg, potentially pathogenic variants and pharmacogenomic variants) and were instructed to research these SNVs as a team using the resources such as dbSNP and OMIM. The research results were then presented as a short PowerPoint presentation, which discussed implications of the sequence variants to the cadaver's pathology. ${ }^{62}$ Learning outcome assessment for this cadaver exome sequencing course has not been reported yet; however, the course learning objectives include the following: students will be able to "describe the benefits and limitations of integrative genomic analyses for patients with inherited and common diseases" and "use online tools to interpret the clinical significance of genomic data." These objectives are on target with the following genomic medicine competencies listed in the genomic testing EPA: 1) discuss the indications for genomic testing - specifically the benefits, risks, and alternatives (the patient care competency); 2) recognize that genomic tests require interpretation with respect to the patient clinical status (the knowledge for practice competency); and 3 ) engage in continuing education regarding advances in genomic medicine and changing indications for and interpretation of genomic testing (the personal and professional development competency). ${ }^{23}$ In addition, the students had opportunities to learn about SNVs affecting drug responses and other phenotypic diversities in populations. This learning experience is applicable to a few of the patient care and knowledge for practice competencies listed in the patient treatment based on genomic results EPA. ${ }^{23}$ Although the hands-on training for bioinformatic analysis was not provided in the cadaver exome sequencing course, the students had a learning experience in clinical reasoning of SNVs. Because the course was offered to the whole MS1 
class with a wide range of educational backgrounds, learning outcomes assessments of the course, when it becomes available, will be highly useful to evaluate the impact of exposing medical students to genomic sequencing as a primer for medical genomics education and its effect for motivating student's lifelong learning of genomic medicine.

\section{Concluding remarks}

The advances in genomic medicine have led many medical schools to reevaluate their genetics curriculum. During this process, active learning modalities have become an essential part of the genetics curriculum, with an emphasis placed on the mastery of practical skills in medical genetics and genomics. Designing such a curriculum equipped with effective learning outcome assessment is challenging; hence, guidelines such as the EPAs in genomic medicine could help by providing succinctly defined competencies in medical genetics and genomics, giving a structural framework that both course content and student assessment can be built off of. The majority of published studies on active learning modules for medical genetics use multiple-choice questions for measuring knowledge gain and self-reported surveys for assessing improved skill levels of students. Because the real mastery of skills in genomic medicine was not assessed by activity-based assessment, judging the efficacy of these learning modules in a clinical setting is difficult. Since genetics instruction has become more integrated with clinical curriculum in recent years, ${ }^{27}$ we are poised to start investigating the effectiveness of active learning modalities for medical students to gain and retain practical skills in genomic medicine using activity-based assessment. The EPAs in genomic medicine ${ }^{23}$ would provide the tools for medical genetics educators to design effective courses and assess the mastery of genomic medicine competencies so that students will be highly functional in the era of genomic medicine.

\section{Acknowledgment}

The author is grateful to Drs Reginald Low and Ezra Amsterdam of the Division of Cardiovascular Medicine at the UC Davis Medical School for their constant support.

\section{Disclosure}

The author reports no conflicts of interest in this work.

\section{References}

1. McBride CM, Wade CH, Kaphingst KA. Consumers' views of directto-consumer genetic information. Annu Rev Genomics Hum Genet. 2010;11:427-446.
2. Powell KP, Cogswell WA, Christianson CA, et al. Primary care physicians' awareness, experience and opinions of direct-to-consumer genetic testing. J Genet Couns. 2012;21(1):113-126.

3. Cichon M, Feldman GL. Opportunities to improve recruitment into medical genetics residency programs: survey results of program directors and medical genetics residents. Genet Med. 2014;16(5):413-418.

4. Cooksey JA, Forte G, Flanagan PA, Benkendorf J, Blitzer MG. The medical genetics workforce: an analysis of clinical geneticist subgroups. Genet Med. 2006;8(10):603-614.

5. Pan V, Yashar BM, Pothast R, Wicklund C. Expanding the genetic counseling workforce: program directors' views on increasing the size of genetic counseling graduate programs. Genet Med.2016;18(8):842-849.

6. Burke W, Emery J. Genetics education for primary-care providers. Nat Rev Genet. 2002;3(7):561-566.

7. Houwink EJF, van Luijk SJ, Henneman L, van der Vleuten C, Dinant GJ, Cornel MC. Genetic educational needs and the role of genetics in primary care: a focus group study with multiple perspectives. $B M C$ Fam Pract. 2011;12:5.

8. Sifri R, Myers R, Hyslop T, et al. Use of cancer susceptibility testing among primary care physicians. Clin Genet. 2003;64(4):355-360.

9. Kemper AR, Trotter TL, Lloyd-Puryear MA, Kyler P, Feero WG, Howell RR. A blueprint for maternal and child health primary care physician education in medical genetics and genomic medicine: recommendations of the United States secretary for health and human services advisory committee on heritable disorders in newborns and children. Genet Med. 2010;12(2):77-80.

10. Haspel RL, Saffitz JE. Genomic oncology education: an urgent need, a new approach. Cancer J. 2014;20(1):91-95.

11. Ho CY, Landstrom AP, Ackerman MJ. Genetics and clinical destiny: improving care in hypertrophic cardiomyopathy. Circulation. 2010;122(23):2430-2440.

12. Burke S, Stone A, Bedward J, Thomas H, Farndon P. A "neglected part of the curriculum" or "of limited use"? Views on genetics training by nongenetics medical trainees and implications for delivery. Genet Med. 2006;8(2):109-115.

13. Harvey EK, Fogel CE, Peyrot M, Christensen KD, Terry SF, McInerney JD. Providers' knowledge of genetics: a survey of 5915 individuals and families with genetic conditions. Genet Med. 2007;9(5):259-267.

14. Bensend TA, Veach PM, Niendorf KB. What's the harm? Genetic counselor perceptions of adverse effects of genetics service provision by non-genetics professionals. J Genet Couns. 2014;23(1):48-63.

15. Brierley KL, Blouch E, Cogswell W, et al. Adverse events in cancer genetic testing medical, ethical, legal, and financial implications. Cancer J. 2012;18(4):303-309.

16. Morgan MA, Driscoll DA, Mennuti MT, Schulkin J. Practice patterns of obstetrician-gynecologists regarding preconception and prenatal screening for cystic fibrosis. Genet Med. 2004;6(5):450-455.

17. Mikat-Stevens NA, Larson IA, Tarini BA. Primary-care providers' perceived barriers to integration of genetics services: a systematic review of the literature. Genet Med. 2014;17(3):169-176.

18. Shields AE, Burke W, Levy DE. Differential use of available genetic tests among primary care physicians in the United States: results of a national survey. Genet Med. 2008;10(6):404-414.

19. Project AoAMCMSO. Contemporary Issues in Medicine: Genetics Education. 2004. Available from: https://members.aamc.org/eweb/ upload/Contemporary\%20Issues\%20in\%20Med\%20Genetics\%20 Education\%20Report\%20VI.pdf. Accessed August 23, 2017.

20. AAMC-HHMI Committee [webpage on the Internet]. Scientific Foundations for Future Physicians. Washington, DC: Association of American Medical Colleges; 2009. Available from: https://members. aamc.org/eweb/DynamicPage.aspx?Action=Add\&ObjectKeyFrom= 1A83491A-9853-4C87-86A4-F7D95601C2E2\&WebCode=ProdDet ailAdd $\&$ DoNotSave $=y e s \&$ ParentObject=CentralizedOrderEntry \&P arentDataObject=Invoice\%20Detail\&ivd_formkey=69202792-63d74ba2-bf4e-a0da41270555\&ivd_prc_prd_key=624AAF22-D6FF-4AA1966B-445C931BCFD2. Accessed August 23, 2017. 
21. Hyland K, Dasgupta S, Garber K. Medical School Core Curriculum in Genetics. Association of Professors of Human and Medical Genetics; 2013. Available from: http://media.wix.com/ugd/3a7b87_7064376a9e b346cfa1b85bc2f137c48f.pdf. Accessed August 23, 2017.

22. Korf B. Genetic and genomic competency in medical practice. Virtual Mentor. 2012;14(8):622-626.

23. Korf BR, Berry AB, Limson M, et al. Framework for development of physician competencies in genomic medicine: report of the Competencies Working Group of the Inter-Society Coordinating Committee for Physician Education in Genomics. Genet Med. 2014;16(11):804-809.

24. Vassy JL, Korf BR, Green RC. How to know when physicians are ready for genomic medicine. Sci Transl Med. 2015;7(287):287fs19.

25. Ten Cate O. Nuts and bolts of entrustable professional activities. J Grad Med Educ. 2013;5(1):157-158.

26. Englander R, Cameron T, Ballard AJ, Dodge J, Bull J, Aschenbrener CA. Toward a common taxonomy of competency domains for the health professions and competencies for physicians. Acad Med. 2013;88(8):1088-1094.

27. Plunkett-Rondeau J, Hyland K, Dasgupta S. Training future physicians in the era of genomic medicine: trends in undergraduate medical genetics education. Genet Med. 2015;17(11):927-934.

28. Frank JR, Snell LS, Cate OT, et al. Competency-based medical education: theory to practice. Med Teach. 2010;32(8):638-645.

29. Issenberg SB, McGaghie WC, Petrusa ER, Lee Gordon D, Scalese RJ. Features and uses of high-fidelity medical simulations that lead to effective learning: a BEME systematic review. Med Teach. 2005;27(1):10-28.

30. Rosen KR, McBride JM, Drake RL. The use of simulation in medical education to enhance students' understanding of basic sciences. Med Teach. 2009;31(9):842-846.

31. Weller JM. Simulation in undergraduate medical education: bridging the gap between theory and practice. Med Educ. 2004;38(1):32-38.

32. Alluri RK, Tsing P, Lee E, Napolitano J. A randomized controlled trial of high-fidelity simulation versus lecture-based education in preclinical medical students. Med Teach. 2016;38(4):404-409.

33. Colleges AoAM [webpage on the Internet]. Medical Simulation in Medical Education: Results of an AAMC Survey. 2011. Available from: https://www.aamc.org/download/259760/data. Accessed March 27, 2017.

34. Freeman S, Eddy SL, McDonough M, et al. Active learning increases student performance in science, engineering, and mathematics. Proc Natl Acad Sci U S A. 2014;111(23):8410-8415.

35. Michael J. Where's the evidence that active learning works? Adv Physiol Educ. 2006;30(4):159-167.

36. Martin BO, Kolomitro K, Lam TC. Training methods A review and analysis. Hum Res Dev Rev. 2014;13(1):11-35.

37. Joyner B, Young L. Teaching medical students using role play: twelve tips for successful role plays. Med Teach. 2006;28(3):225-229.

38. Nestel D, Tierney T. Role-play for medical students learning about communication: guidelines for maximising benefits. BMC Med Educ. 2007;7:3.

39. Bosse HM, Nickel M, Huwendiek S, Junger J, Schultz JH, Nikendei C. Peer role-play and standardised patients in communication training: a comparative study on the student perspective on acceptability, realism, and perceived effect. BMC Med Educ. 2010;10:27.

40. Nikendei C, Zeuch A, Dieckmann P, et al. Role-playing for more realistic technical skills training. Med Teach. 2005;27(2):122-126.

41. Schlegel C, Woermann U, Shaha M, Rethans JJ, van der Vleuten C. Effects of communication training on real practice performance: a role-play module versus a standardized patient module. J Nurs Educ. 2012;51(1):16-22.

42. Rao D. Skills development using role-play in a first-year pharmacy practice course. Am J Pharm Educ. 2011;75(5):84.
43. McIlvried DE, Prucka SK, Herbst M, Barger C, Robin NH. The use of role-play to enhance medical student understanding of genetic counseling. Genet Med. 2008;10(10):739-744.

44. Xu XF, Wang Y, Wang YY, Song M, Xiao WG, Bai Y. Role-playing is an effective instructional strategy for genetic counseling training: an investigation and comparative study. BMC Med Educ. 2016;16(1): 235.

45. Alfes CM. Nursing alumni as standardized patients: an untapped resource. Clin Simul Nurs. 2013;9(12):E593-E597.

46. Mesquita AR, Lyra DP, Brito GC, Balisa-Rocha BJ, Aguiar PM, Neto ACD. Developing communication skills in pharmacy: a systematic review of the use of simulated patient methods. Patient Educ Couns. 2010;78(2):143-148.

47. Nestel D, Clark S, Tabak D, et al. Defining responsibilities of simulated patients in medical education. Simul Healthc. 2010;5(3):161-168.

48. McGovern MM, Johnston M, Brown K, Zinberg R, Cohen D. Use of standardized patients in, undergraduate medical genetics education. Teach Learn Med. 2006;18(3):203-207.

49. Metcalf MP, Tanner TB, Buchanan A. Effectiveness of an online curriculum for medical students on genetics, genetic testing and counseling. Med Educ Online. 2010;15.

50. Makransky G, Bonde MT, Wulff JS, et al. Simulation based virtual learning environment in medical genetics counseling: an example of bridging the gap between theory and practice in medical education. BMC Med Educ. 2016;16:98.

51. Smith HC. A course director's perspectives on problem-based learning curricula in biochemistry. Acad Med. 2002;77(12 pt 1):1189-1198.

52. Bean LJ, Fridovich-Keil J, Hegde M, Rudd MK, Garber KB. The virtual diagnostic laboratory: a new way of teaching undergraduate medical students about genetic testing. Genet Med. 2011;13(11):973-977.

53. Sharp RR, Goldlust ME, Eng C. Addressing gaps in physician education using personal genomic testing. Genet Med. 2011;13(8):750-751.

54. Garber KB, Hyland KM, Dasgupta S. Participatory genomic testing as an educational experience. Trends Genet. 2016;32(6):317-320.

55. Walt DR, Kuhlik A, Epstein SK, et al. Lessons learned from the introduction of personalized genotyping into a medical school curriculum. Genet Med. 2011;13(1):63-66.

56. Linderman MD, Bashir A, Diaz GA, et al. Preparing the next generation of genomicists: a laboratory-style course in medical genomics. $B M C$ Med Genomics. 2015;8:47.

57. Salari K, Karczewski KJ, Hudgins L, Ormond KE. Evidence that personal genome testing enhances student learning in a course on genomics and personalized medicine. PLoS One. 2013;8(7):e68853.

58. Sanderson SC, Linderman MD, Zinberg R, et al. How do students react to analyzing their own genomes in a whole-genome sequencing course?: outcomes of a longitudinal cohort study. Genet Med. 2015;17(11):866-874.

59. Vernez SL, Salari K, Ormond KE, Lee SS. Personal genome testing in medical education: student experiences with genotyping in the classroom. Genome Med. 2013;5(3):24.

60. Sanderson SC, Linderman MD, Kasarskis A, et al. Informed decisionmaking among students analyzing their personal genomes on a whole genome sequencing course: a longitudinal cohort study. Genome Med. 2013;5(12):113.

61. Gerhard GS, Paynton B, Popoff SN. Integrating cadaver exome sequencing into a first-year medical student curriculum. JAMA. 2016;315(6):555-556.

62. Gerhard GS, Jin Q, Paynton BV, Popoff SN. The Anatomy to Genomics (ATG) start genetics medical school initiative: incorporating exome sequencing data from cadavers used for anatomy instruction into the first year curriculum. BMC Med Genomics. 2016;9(1):62. 
Advances in Medical Education and Practice

Dovepress

\section{Publish your work in this journal}

Advances in Medical Education and Practice is an international, peerreviewed, open access journal that aims to present and publish research on Medical Education covering medical, dental, nursing and allied health care professional education. The journal covers undergraduate education, postgraduate training and continuing medical education including emerging trends and innovative models linking education, research, and health care services. The manuscript management system is completely online and includes a very quick and fair peer-review system. Visit http://www.dovepress.com/testimonials.php to read real quotes from published authors.

Submit your manuscript here: http://www.dovepress.com/advances-in-medical-education-and-practice-journal 